\title{
Collaborate for Healthy Weight
}

\author{
Interview with Charles J. Homer, M.D., M.P.H., \\ Chief Executive Officer of the National Initiative for Children's Healthcare Quality
}

$\mathrm{C}$ ollaborate for Healthy Weight is a project from the National Initiative for Children's Healthcare Quality (NICHQ) and the Health Resources and Services Administration (HRSA), funded by the Affordable Care Act's Prevention and Public Health Fund. The aim of the project is to bring together primary care, public health, and community organizations to discover sustainable ways to promote healthy weight and eliminate disparities in weight status in communities across the United States. The project, which focuses on disadvantaged and underserved populations, has just entered its second phase with the addition of 40 new teams to its initial cohort of 10. Hear from Dr. Charles Homer on the progress of the project as well as what's in store for Phase Two.

Collaborate for Healthy Weight is a program in which you select local teams from around the country and assist them with their initiatives geared toward community health. First off, could you give us an example of a team you've been working with?

Dr. Homer: There are two teams that would be good to highlight here - one on each coast of the country. First is the San Diego Healthy Weight Collaborative, based primarily in Chula Vista in San Diego County. They have identified a subset of the overall County (adult and pediatric residents of the South Bay area) that has particularly high need. They serve a demanding population with very high rates of obesity.

As we have encouraged all of the community teams, they have identified a population of focus. Their population is served at a community health center, at a school, and early childhood program. They have adopted a common message to use across all those settings. Like many of the teams, they have adopted the 5-2-1-0 messaging ( 5 fruits and vegetables per day, 2 hours or less of screen time, 1 hour of physical activity, and 0 sugary drinks). And then they went on to test that message. They have done focus groups in multiple settings to make sure that the message is effective and understood, which I think is extremely helpful.

Building on their message, they are driving change in each of the settings in an integrated and coordinated way.
For example, within the schools they have assisted in revising wellness policy consistent with the messaging and consistent with the concept of each individual child having an understanding of their own weight and having a healthy weight plan. Also they ensure that other wellness policies in the school, such as quality of school lunches, are consistent with that message and framework.

They are also using a very similar approach in the early childhood settings. They have done focus groups and developed wellness policy in the early childhood programs. They have helped the early childhood center that they work with to develop practices that are consistent with that policy. And then, similarly, in the clinical setting, they have reviewed both the wellness policy within the community health center and the clinical parameters of assessing BMI and providing appropriate counseling for kids.

So what I really like is that they have taken the same message and have used it to create an integrated approach across many different segments and sectors of their community. They have a coalition in that community that is really thinking about their population of focus and developing a common approach and message across multiple settings.

San Diego County had already done a great deal to address obesity before joining our initiative. Sarasota County in Florida joined our program much earlier on in the development of their work on this topic, and that is why I highlight it. Although many may think of Sarasota as a fairly affluent community, it certainly has a significant level of poverty and significant levels of obesity.

They have used Collaborate for Healthy Weight to help build a strong coalition and bring in multiple partners. They have used our measurement strategy as a score card, which they have posted in their health department. Like San Diego, they have adopted the 5-2-1-0 messaging. Sarasota County schools have really jumped onto this program and are incorporating a variety of elements into their activities. We are really excited about the pace in Sarasota County. They started at a very different level from San Diego, but the pace of their change is very exciting. 
Both San Diego and Sarasota Counties were part of the initial cohort of 10 teams in the program, correct?

Dr. Homer: Yes, and the idea with the initial 10 was we wanted to have teams that we thought could hit the ground running. We wanted to learn as much as we could from them so that we could spread those lessons to the broader set of communities with whom we have just started to work.

The broader set of communities you mention is part of the second phase of the Collaborate for Healthy Weight project-with an introduction of 40 new teams. How has the project handled the large increase in teams?

Dr. Homer: With the next round, there are really two important challenges. One, it has obviously greatly increased in scale. The other, in part because of the scale and in part to be efficient in our use of resources, is that we are conducting the activities of this larger program virtually. With the first 10 , we brought them together for learning sessions (face-to-face meetings) and provided in-person training on both obesity content-addressing obesity, obesity prevention, and treatment - and quality improvement. And we did a lot of face-to-face coaching. The first group of teams will have the opportunity to do that twice, in addition to one virtual training and coaching activity.

In the second round with 40 teams, we are doing all of the training and coaching virtually. We have developed and refined our Collaborate for Healthy Weight website. We have also built out an extranet site called the Improvement Lab with a great deal of resources for these teams. We are creating asynchronous video-training opportunities so the teams can learn about obesity and quality improvement on their own time. Also we will have a variety of online synchronous activities; that is when all of the teams will be online together, sharing ideas, experiences, etc., through chat functions and video conference calls.

We are also using social media tools, Twitter and Facebook in particular, and that is very much part of the strategy. And then we have built up some tools from our previous work and from the first 10 teams, as well as from our faculty. So there are resources such as healthy weight plans and coalition self-assessment tools.

In addition, we are creating some more human support for them, much of which will still be virtual-conference and individual phone calls - and some of which may be by visits. And those include mentoring from the first 10 teams, who will be providing support to the newly joined group.

Also we have built out regional faculty to provide additional support. The HRSA is not only helping fund this work; they are working hand-in-hand with us. HRSA has regional offices around the country and we have been providing training and support to those regional offices so that they will be able to provide technical assistance to teams within their geographic region.
It seems as if one of the benefits of getting all the teams on this platform is that they can speak the same language with regard to their projects and goals. Is that something that you are finding?

Dr. Homer: A key part of all of NICHQ's improvement work is collaborative learning. In everything we do, and certainly in this program, we expect there to be at least as much learning as there is teaching between the teams. The phrase we use is: "All teach, all learn." And that, in fact, does happen in all of our programs. So we certainly expect that to happen in this project.

With this idea in mind, there is actually an advantage in having more teams, because there are more chances for learning. Some people call it "trial and error." Other people may call it "trial and learning." So, if there are 40 teams trying different things, there is actually more opportunity for rapid learning if they share with each other and do not repeat the same challenges, or mistakes, that other teams might have made.

We have been discussing video and collaborative tools available to the teams on the virtual learning site. Are there any other resources there that you would like to highlight?

Dr. Homer: We certainly make available a broad variety of tools and materials. And we have not felt the need to recreate existing tools. So, for example, the CDC has great sets of tools and we make those readily available.

One of the things that we are sharing too is making sure that the Phase Two teams have examples of the Phase One action plans and aim statements. Those are available on the project extranet. We want all users to be able to learn from what the teams already put together to see how they can tweak it for their own community.

\section{What thoughts can be shared about effective means of taking what works and making sure it spreads appropriately? How do we avoid the inefficiencies of reinventing the same wheels?}

Dr. Homer: Well, one way we have helped filter some of the resources out there is by creating a comprehensive resource we call a "Change Package," which is really a listing of recommendations that we, the published literature, or the consensus of experts, found effective. We realized, though, that the volume of materials and recommendations we were providing was potentially overwhelming so we boiled that down to what we call an "Implementation Guide," which is a set of six specific strategies and then some particular tools that can facilitate those strategies.

The strategies include: Committing to develop and implement a community action plan; developing consistent messaging around healthy weight; assessing weight status through the population; applying and using a healthy weight plan; building the capacity in the community to meet the needs of the population; and then improv- 
ing the environment to promote healthy weight largely through policy change. And each of those, of course, has more detail behind it.

Additionally, I have long had the vision, which we have not yet fully realized, in which there would be the opportunity for people to come and rate a variety of the resources - similar to the kinds of applications like Yelp ${ }^{\circledR}$ or OpenTable ${ }^{\circledR}$, for example.

So, that is how we try to help boil down the vast array of resources out there. People have found the provision of the Implementation Guide extremely helpful - the idea of simplifying and sorting and categorizing activities.

In terms of getting the message out there, we have committed in this project-different from many traditional quality improvement projects - to getting the word out as quickly as we can to as broad an audience as we can. For example, we have regular "Action Calls" where we both share the results of what we are learning in the project and highlight a particular topic that is relevant to the work that the teams are doing. Our most recent Action Call focused on effective coalition building, for example.

In addition we have a publicly facing Web site (www. collaborateforhealthyweight.org) that shares on an ongoing basis the lessons from the teams and will be a vehicle for disseminating the tools and materials.

\section{Are the Action Calls available on the publicly facing site?}

Dr. Homer: Yes, those are definitely open to the public. They are available under the "Resources" tab on the Collaborate for Healthy Weight site.

\section{How do you evaluate the progress and effective- ness of the project? How do you know that positive change/improvement is being achieved?}

Dr. Homer: Measurement is very important to our work. And we evaluate the progress on multiple levels. In all of our quality improvement work as an organization, we always ask that teams participating in our programs measure their own progress and report it to us and share with other teams. We also ask that they look at it themselves and reflect on what they are doing and try to continuously make it better. That is a core characteristic of quality improvement work.

In this program, in particular, we have established a set of measures for these teams to use, and we are simplifying the measures somewhat for the second round, but the same general themes apply. The measuresgiven the short time that we are working with the teams, which is really less than a year-mainly focus on measuring the processes: Are teams taking the necessary actions to change the weight curve in their communities?

We believe that having a strong effective coalition is critical to having these communities experience change. And we have identified what we believe is a very good measure of coalition function, which is the Wilder Collaboration Factors Inventory. We have the teams repeatedly assess themselves over time to see if their coalition is getting stronger.

We have asked the teams to identify a message and to disseminate that message to a specified population. Then we ask them to measure the extent to which that message is reaching the population on which they are focusing.

I'd like to really highlight the aspect of identifying a particular population. We think one of the most important differences of this work, compared to a lot of other excellent work that people are doing to address obesity, is we ask these teams to identify the population on which they are focusing and to think about what that population needs in terms of either prevention or treatment or both; then have multiple sectors come together to plan and implement those changes.

The reason I bring up the population of focus with the measurement discussion is that measurement is a critical step in establishing clarity around the population of focus. So, we can ask: Do you have a message? Is the message getting out there? Is the message getting to everybody in clinical practices? Does everybody within that target population have an understanding of their weight status, and whether they are in the healthy weight zone? Do they have a healthy weight plan?

We do employ some of the more traditional measures that are used in clinical settings, such as whether both adults and children have had their BMI assessed and receive appropriate counseling about nutrition and physical activity. Then we also try to measure the extent to which policies are moving forward and are being put into place, such as a school wellness policy or a health center wellness policy.

\section{Does the specificity of the population of focus vary between projects?}

Dr. Homer: Some of the populations are as small as a single middle school or an early childhood program or people cared for at one community health center. And some are broader - a neighborhood, a city, a county. And we have been fine with that. Though, my sense is some of the initial teams started very broad and then narrowed their focus so that they could be more strategic.

\section{What are your main goals for the next phase of the project?}

Dr. Homer: In this project, it is very important that we help these communities establish effective coalitions and make real, positive changes. We want the teams to get some early successes in making these changes and then build on them. What we are trying to do, just like for the children the teams are affecting, is help put the community teams on the right trajectory. We are trying to get these teams on a sustainable trajectory for community improvement. That is our primary goal. 
Our secondary goal is to create enduring infrastructure with this virtual technology that will allow us to support not just the 40 teams, but also a broader community across the country. If we can demonstrate an effective methodology for reaching large numbers of communities, we would be achieving what we set out to do over the course of the project.

Could you speak more broadly about childhood obesity in disadvantaged communities? Do you see real change occurring from the initiative?

Dr. Homer: The obesity epidemic affects the whole country, and it affects children across classes and racial groups. It is particularly accentuated in disadvantaged communities so that is why we are focusing this initiative there. We think equity is a very important issue and, particularly, if you look at the tail end of the distribution of the weight curve and the kids who are over the 99th percentile in weight, there is a very substantial disparate impact in disadvantaged communities. Over their lifetimes, this will have a potentially devastating effect in terms of increased risks of diabetes and cardiovascular disease, and really can impair people's ability to move out of poverty and have productive jobs and familiesbecause weight is a very important predictor of people's ability to lead productive and healthy lives.

So, that is why we are focusing on those communities. And the challenges in those communities are even greater in the sense of: Do people even have access to healthy foods? Can they afford them? Can you prepare healthy foods in a reasonable amount of time if you are working multiple low-paid jobs? Are kids able to play on the streets? Do the kids have safe playgrounds to go to? Are there afterschool programs? I could go on.

In addition, at the poverty level, there is increasing evidence about the impact of multiple stressors on families and the biologic impact that has on the way children metabolize. So again, there are multiple factors that affect disadvantaged communities that we think merit our attention.

Do I see change happening in those communities? Despite the breadth and depth of this epidemic, I do think the country is mobilized around this work. I think again, from the White House on down and from the communities on up, this is an epidemic about which people are aware.

For example, in other topical areas, when we talk to physicians, they may not be aware of the issue at hand. We might say: "If you follow this protocol, your immunization rate is going to improve. Your asthma management could be better." And the clinicians may feel that they are already doing a good job there and to convince them to change is challenging.

In this case, it is not hard to get people engaged. Any clinician who has been out there for 10 or 15 years can see that their population has changed. They can see that there are children who are overweight, and getting the clinicians to focus on improvement is an easy sell. And I think it is the same thing for the general public. So the country is mobilized and interested in addressing this topic. And we are focusing on the disadvantaged communities because that is where the epidemic is the worst, and they are the communities that can benefit the most from the additional resources and coaching that we can provide.

— Jamie Devereaux, Features Editor 